\title{
A CHARACTERIZATION OF STRICT JACOBI-NIJENHUIS MANIFOLDS THROUGH THE THEORY OF LIE ALGEBROIDS
}

\author{
J. M. Nunes DA CosTA* \\ Departamento de Matemática, Universidade de Coimbra \\ Apartado 3008, 3001-454 Coimbra, Portugal \\ (e-mail: jmcosta@mat.uc.pt)
}

(Received January 8, 2002 - Revised March 11, 2002)

\begin{abstract}
We obtain a characterization of strict Jacobi-Nijenhuis structures using the equivalent notions of generalized Lie bialgebroid and Jacobi bialgebroid.
\end{abstract}

Keywords: Jacobi-Nijenhuis manifold, Poisson-Nijenhuis manifold, Lie algebroid, Lie bialgebroid.

\section{Introduction}

The notion of Jacobi-Nijenhuis manifold was introduced in [12] by J. C. Marrero, J. Monterde and E. Padrón as a generalization of the weak Poisson-Nijenhuis structure presented in [13]. In this work we introduce the notion of strict Jacobi-Nijenhuis manifold, which seems to be the natural generalization of the definition of PoissonNijenhuis manifold initially given by F. Magri and C. Morosi in [11].

When a Poisson manifold $(M, \Lambda)$ is equipped with a Nijenhuis tensor $N$, we can associate with this manifold two Lie algebroid structures defined respectively on the tangent and on the cotangent bundles of $M$. Using the notion of Lie bialgebroid, which was introduced by $\mathrm{K}$. Mackenzie and $\mathrm{P}$. Xu in [10], Y. Kosmann-Schwarzbach showed in [7] that $(M, \Lambda, N)$ is a Poisson-Nijenhuis manifold if and only if these two Lie algebroids constitute a Lie bialgebroid. Our aim is to show that a similar relation can be obtained when a differentiable manifold is equipped with a Jacobi structure and a Nijenhuis operator. For this purpose, we will use the notion of generalized Lie bialgebroid, introduced by D. Iglesias and J. C. Marrero in [2]. This notion is equivalent to the one introduced by J. Grabowski and G. Marmo in [1], under the name of Jacobi bialgebroid. Generalized Lie bialgebroids are closely related to Jacobi structures. In fact, it was proved in [2] that with each Jacobi manifold one can associate, in a certain manner, a generalized Lie bialgebroid and that the base manifold of a generalized Lie bialgebroid possesses a Jacobi structure.

Similar results to those found in this paper were obtained, independently, in [3].

* Supported by CMUC-FCT and PRAXIS.

Presented during the conference "Multi-Hamiltonian Structures: Geometric and Algebraic Aspects", 9-17 August 2001, in Mathematical Conference Center in Będlewo, Poland. 


\section{Lie bialgebroids and Poisson-Nijenhuis manifolds}

A Lie algebroid $(A,[],, \rho)$ over a manifold $M$ is a vector bundle $A$ over $M$ together with a bundle map $\rho: A \rightarrow T M$ and a Lie algebra structure $[$,$] on the$ space $\Gamma(A)$ of the global cross sections such that

(i) the map $\Gamma(\rho): \Gamma(A) \rightarrow \mathfrak{X}(M)$, induced by $\rho$, is a Lie algebra homomorphism;

(ii) for any $f \in C^{\infty}(M)$ and $X, Y \in \Gamma(A)$,

$$
[X, f Y]=f[X, Y]+(\Gamma(\rho)(X) . f) Y .
$$

The map $\rho$ is called the anchor map and usually $\Gamma(\rho)$ is denoted by $\rho$.

It is well known [8] that with each Lie algebroid $(A,[],, \rho)$ a differential $d$ on the graded space of sections of $\Lambda A^{*}=\oplus_{k \in \mathbb{Z}} \Lambda^{k} A^{*}$ is associated, where $A^{*}$ is the dual vector bundle of $A$. More precisely, $d$ is a derivation of degree 1 and of square 0 of the associative graded commutative algebra $\left(\Gamma\left(\Lambda A^{*}\right), \wedge\right)$. Also the Lie bracket on $\Gamma(A)$ can be extended to the algebra of sections of $\Lambda A, \Gamma(\Lambda A)=\oplus_{k \in \mathbb{Z}} \Gamma\left(\Lambda^{k} A\right)$. The result is a graded Lie bracket [,] which is called the Schouten bracket of the Lie algebroid.

Suppose that the vector bundle $(A,[],, \rho)$ and its dual vector bundle $\left(A^{*},[,]_{*}, \rho_{*}\right)$ are both Lie algebroids over a manifold $M$. Let $d$ (resp. $d_{*}$ ) denote the differential of $A$ (resp. $\left.A^{*}\right)$. The pair $\left(A, A^{*}\right)$ is a Lie bialgebroid [10] if for all $X, Y \in \Gamma(A)$,

$$
d_{*}[X, Y]=\left[d_{*} X, Y\right]+\left[X, d_{*} Y\right] .
$$

(Equivalently, $\left(A, A^{*}\right)$ is a Lie bialgebroid if $d_{*}$ is a derivation of $(\Gamma(\Lambda A)$, [, ]), see [6]).

This notion is self-dual, in the sense that if $\left(A, A^{*}\right)$ is a Lie bialgebroid so is $\left(A^{*}, A\right)$, cf. $[6,10]$.

Example 1. Let $(M, \Lambda)$ be a Poisson manifold and $\Lambda^{\sharp}: T^{*} M \rightarrow T M$ the vector bundle morphism given by $\left\langle\beta, \Lambda^{\sharp}(\alpha)\right\rangle=\Lambda(\alpha, \beta)$ for all 1-forms $\alpha$ and $\beta$ in $M$. Then the pair $\left(\left(T^{*} M,[,]_{\Lambda}, \Lambda^{\sharp}\right),\left(T M,[],, \mathrm{Id}_{T M}\right)\right)$ is a Lie bialgebroid over $M$, where $[,]_{\Lambda}$ is the Lie bracket of 1 -forms given, for all $\alpha, \beta \in \Omega^{1}(M)$, by

$$
[\alpha, \beta]_{\Lambda}=\mathcal{L}_{\Lambda^{\sharp}(\alpha)} \beta-\mathcal{L}_{\Lambda^{\sharp}(\beta)} \alpha-d(\Lambda(\alpha, \beta)) .
$$

The differential of $\left(T M,[],, \mathrm{Id}_{T M}\right)$ is the de Rham differential, while the differential of $\left(T^{*} M,[,]_{\Lambda}, \Lambda^{\sharp}\right)$ is the Lichnerowicz-Poisson differential $d_{\Lambda}=[\Lambda,$.

The previous example shows a relation between Poisson manifolds and Lie bialgebroids. Another link relating these two structures is the following [10]: if $\left(A, A^{*}\right)$ is a Lie bialgebroid over $M$, there exists on $M$ an induced Poisson structure,

$$
\{f, h\}=\left\langle d f, d_{*} h\right\rangle, \quad f, h \in C^{\infty}(M) .
$$

DEFINITION 1. [11] A Poisson-Nijenhuis manifold $(M, \Lambda, N)$ is a Poisson manifold $(M, \Lambda)$ equipped with a tensor field $N$ of type $(1,1)$ with vanishing Nijenhuis torsion, i.e. a Nijenhuis tensor, satisfying the following compatibility conditions: 
(i) $N \Lambda^{\sharp}=\Lambda^{\sharp} .{ }^{t} N$ and

(ii) $C(\Lambda, N)=0$, where

$$
C(\Lambda, N)(\alpha, \beta)=[\alpha, \beta]_{N \Lambda}-\left[{ }^{t} N \alpha, \beta\right]_{\Lambda}-\left[\alpha,{ }^{t} N \beta\right]_{\Lambda}+{ }^{t} N[\alpha, \beta]_{\Lambda},
$$

for all $\alpha, \beta \in \Omega^{1}(M),{ }^{t} N$ stands for the transpose of $N$ and [, $]_{\Lambda}$ (resp. [, $]_{N \Lambda}$ ) is the bracket (2) associated with $\Lambda$ (resp. $N \Lambda$ ).

We should remark that condition (ii) of Definition 1 can be weakened, as it was done in [13], to obtain the so-called weak Poisson-Nijenhuis manifold.

It is well known [8] that when $N$ is a Nijenhuis tensor on $M$, the triple $\left(T M,[,]_{N}, N\right)$ is a Lie algebroid, where $[,]_{N}$ is given by

$$
[X, Y]_{N}=[N X, Y]+[X, N Y]-N[X, Y], \quad X, Y \in \mathfrak{X}(M) .
$$

The next theorem gives a characterization of Poisson-Nijenhuis manifolds using the notion of Lie bialgebroid.

THEOREM 1. [7] Let $(M, \Lambda)$ be a Poisson manifold and $N$ a Nijenhuis tensor on $M$. Then $(M, \Lambda, N)$ is a Poisson-Nijenhuis manifold if and only if the pair

$$
\left(\left(T M,[,]_{N}, N\right),\left(T^{*} M,[,]_{\Lambda}, \Lambda^{\sharp}\right)\right)
$$

is a Lie bialgebroid.

\section{Jacobi bialgebroids and Jacobi manifolds}

We recall that a Jacobi structure on a manifold $M$ is a pair $(\Lambda, E)$, where $\Lambda$ is a bivector and $E$ is a vector field such that $[\Lambda, \Lambda]=-2 E \wedge \Lambda$ and $[E, \Lambda]=0$.

Let $(M, \Lambda, E)$ be a Jacobi manifold. Denote by $(\Lambda, E)^{\#}: T^{*} M \times \mathbb{R} \rightarrow T M \times \mathbb{R}$ the vector bundle morphism given by $(\Lambda, E)^{\#}(\alpha, f)=\left(\Lambda^{\#}(\alpha)+f E,-\langle\alpha, E\rangle\right)$, for any section $\alpha$ of $T^{*} M$ and $f \in C^{\infty}(M)$. In opposition to the case of a Poisson manifold, in general one cannot define a Lie algebroid structure on the cotangent bundle of a Jacobi manifold. However, in [5] it was shown that if $(M, \Lambda, E)$ is a Jacobi manifold, then $\left(T^{*} M \times \mathbb{R},[,]_{(\Lambda, E)}, \pi \circ(\Lambda, E)^{\sharp}\right)$ is a Lie algebroid over $M$, where $\pi: T M \times \mathbb{R} \rightarrow T M$ is the projection over the first factor and $[,]_{(\Lambda, E)}$ is the bracket given by

$$
[(\alpha, f),(\beta, h)]_{(\Lambda, E)}:=(\gamma, r),
$$

with

$$
\begin{aligned}
\gamma:= & \mathcal{L}_{\Lambda^{*}(\alpha)} \beta-\mathcal{L}_{\Lambda^{*}(\beta)} \alpha-d(\Lambda(\alpha, \beta))+f \mathcal{L}_{E} \beta-h \mathcal{L}_{E} \alpha-i_{E}(\alpha \wedge \beta), \\
& r:=-\Lambda(\alpha, \beta)+\Lambda(\alpha, d h)-\Lambda(\beta, d f)+\langle f d h-h d f, E\rangle .
\end{aligned}
$$

The associated differential $d_{*}$ is given for all $(P, Q) \in \mathcal{V}^{k}(M) \oplus \mathcal{V}^{k-1}(M)$ by [4]

$$
d_{*}(P, Q)=([\Lambda, P]+k E \wedge P+\Lambda \wedge Q,-[\Lambda, Q]+(1-k) E \wedge Q+[E, P]),
$$

where $\mathcal{V}^{k}(M)=\Gamma\left(\Lambda^{k}(T M)\right)$. 
On the other hand, if $M$ is a differentiable manifold, then the triple ( $T M \times$ $\mathbb{R},[],, \pi)$ is a Lie algebroid over $M$, where $\pi$ is the projection over the first factor and $[$,$] is given by$

$$
[(X, f),(Z, h)]=([X, Z], X \cdot h-Z \cdot f), \quad(X, f),(Z, h) \in \mathfrak{X}(M) \times C^{\infty}(M) .
$$

The associated differential is $\mathrm{d}=(d,-d), d$ being the de Rham differential.

When $(M, \Lambda, E)$ is a Jacobi manifold, a natural question that arises is whether the pair $\left(T^{*} M \times \mathbb{R}, T M \times \mathbb{R}\right)$ is a Lie bialgebroid. The answer is no! This situation motivated the introduction, by D. Iglesias and J. C. Marrero [2], of the generalized Lie bialgebroids. The definition of generalized Lie bialgebroid was recently recast in simpler terms by J. Grabowski and G. Marmo in [1], under the name of Jacobi bialgebroid.

Let $(A,[],, \rho)$ be a Lie algebroid over $M$ and $\theta \in \Gamma\left(A^{*}\right)$ a 1-cocycle for the Lie algebroid cohomology complex with trivial coefficients (see [9]), i.e. for all $X, Z \in \Gamma(A)$,

$$
\theta([X, Z])=\rho(X) .(\theta(Z))-\rho(Z) .(\theta(X)) .
$$

Using the 1-cocycle $\theta$, we can define a new representation $\rho^{\theta}$ of the Lie algebra $(\Gamma(\Lambda),[]$,$) on C^{\infty}(M)$, by setting

$$
\rho^{\theta}: \Gamma(A) \times C^{\infty}(M) \rightarrow C^{\infty}(M), \quad(X, f) \mapsto \rho^{\theta}(X, f)=\rho(X) . f+\theta(X) f .
$$

Therefore, we obtain a new cohomology complex, whose differential cohomology operator is given by

$$
d^{\theta}: \Gamma\left(\Lambda^{k} A^{*}\right) \rightarrow \Gamma\left(\Lambda^{k+1} A^{*}\right), \quad \beta \mapsto d^{\theta}(\beta)=d \beta+\theta \wedge \beta .
$$

Also, for any $X \in \Gamma(A)$, the Lie derivative operator with respect to $X$ is given by

$$
\mathcal{L}_{X}^{\theta}: \Gamma\left(\Lambda^{k} A^{*}\right) \rightarrow \Gamma\left(\Lambda^{k} A^{*}\right), \quad \beta \mapsto \mathcal{L}_{X}^{\theta}(\beta)=\mathcal{L}_{X} \beta+\theta(X) \beta .
$$

It is also possible to consider a $\theta$-Schouten bracket on the graded algebra $\Gamma(\Lambda A)$, denoted by $[,]^{\theta}$, which is defined as follows:

$$
\begin{aligned}
& {[,]^{\theta}: \Gamma\left(\Lambda^{p} A\right) \times \Gamma\left(\Lambda^{q} A\right) \rightarrow \Gamma\left(\Lambda^{p+q-1} A\right)} \\
& \quad(P, Q) \mapsto[P, Q]^{\theta}=[P, Q]+(p-1) P \wedge\left(i_{\theta} Q\right)+(-1)^{p}(q-1)\left(i_{\theta} P\right) \wedge Q .
\end{aligned}
$$

Suppose that $(A,[],, \rho)$ is a Lie algebroid over $M$ such that in the dual bundle $A^{*}$ of $A$ there also exists a Lie algebroid structure over $M,\left([,]_{*}, \rho_{*}\right)$. Let $\theta \in \Gamma\left(A^{*}\right)$ (resp. $W \in \Gamma(A)$ ) be a 1-cocycle in the Lie algebroid cohomology complex of $(A,[],, \rho)$ (resp. $\left.\left(A^{*},[,]_{*}, \rho_{*}\right)\right)$.

DEFINITION 2. [2] The pair $\left((A, \theta),\left(A^{*}, W\right)\right)$ is a generalized Lie bialgebroid if for all $X, Z \in \Gamma(A)$ and $P \in \Gamma\left(\Lambda^{p} A\right)$, 
1. $d_{*}^{W}[X, Z]=\left[d_{*}^{W} X, Z\right]^{\theta}+\left[X, d_{*}^{W} Z\right]^{\theta}$;

2. $\left(\mathcal{L}_{*}^{W}\right)_{\theta}(P)+\left(\mathcal{L}^{\theta}\right)_{W}(P)=0$.

DEFINITION 3. [1] The pair $\left((A, \theta),\left(A^{*}, W\right)\right)$ is a Jacobi bialgebroid if for all $P \in \Gamma\left(\Lambda^{p} A\right)$ and $Q \in \Gamma(\Lambda A)$,

$$
d_{*}^{W}[P, Q]^{\theta}=\left[d_{*}^{W} P, Q\right]^{\theta}+(-1)^{p+1}\left[P, d_{*}^{W} Q\right]^{\theta} .
$$

The equivalence of Definitions 2 and 3 was proved in [1]. Consequently, generalized Lie bialgebroids and Jacobi bialgebroids designate exactly the same structure. When $\theta=0$ and $W=0$, the Jacobi bialgebroid is a Lie bialgebroid.

Let $(M, \Lambda, E)$ be a Jacobi manifold and let us consider the two Lie algebroids $\left(T^{*} M \times \mathbb{R},[,]_{(\Lambda, E)}, \pi \circ(\Lambda, E)^{\sharp}\right)$ and $(T M \times \mathbb{R},[],, \pi)$ mentioned above. Then $\theta=$ $(0,1)$ (resp. $W=(-E, 0)$ ) is a 1-cocycle of $T M \times \mathbb{R}$ (resp. $T^{*} M \times \mathbb{R}$ ) and the pair $\left((T M \times \mathbb{R}, \theta),\left(T^{*} M \times \mathbb{R}, W\right)\right)$ is a Jacobi bialgebroid.

Similary to the relation between Lie bialgebroids and Poisson manifolds, whenever $\left((A, \theta),\left(A^{*}, W\right)\right)$ is a Jacobi bialgebroid over $M$, there exists on $M$ an induced Jacobi structure given by [2]:

$$
\{f, h\}=\left\langle d^{\theta} f, d_{*}^{W} h\right\rangle, \quad f, h \in C^{\infty}(M) .
$$

\section{Jacobi bialgebroids and strict Jacobi-Nijenhuis manifolds}

Let $M$ be a $C^{\infty}$-differentiable manifold and $\mathcal{N}: \mathfrak{X}(M) \times C^{\infty}(M) \rightarrow \mathfrak{X}(M) \times$ $C^{\infty}(M)$ a $C^{\infty}(M)$-linear map defined, for all $(X, f) \in \mathfrak{X}(M) \times C^{\infty}(M)$, by

$$
\mathcal{N}(X, f)=(N X+f Y,\langle\gamma, X\rangle+g f),
$$

where $N$ is a tensor field of type $(1,1)$ on $M, Y \in \mathfrak{X}(M), \gamma \in \Omega^{1}(M)$ and $g \in$ $C^{\infty}(M) . \mathcal{N}:=(N, Y, \gamma, g)$ can be considered as a vector bundle map, $\mathcal{N}: T M \times \mathbb{R} \rightarrow T M \times \mathbb{R}$. We may define the Nijenhuis torsion $\mathcal{T}(\mathcal{N})$ of $\mathcal{N}$ with respect to the Lie bracket $(7)$. When $\mathcal{T}(\mathcal{N})$ vanishes identically, we say that $\mathcal{N}$ is a Nijenhuis operator on $M$.

Suppose now that $M$ is equipped with a Jacobi structure $(\Lambda, E)$ and a Nijenhuis operator $\mathcal{N}$ and consider a tensor field $\Lambda_{1}$ of type $(2,0)$ and a vector field $E_{1}$ on $M$, defined by

$$
\left(\Lambda_{1}, E_{1}\right)^{\#}=\mathcal{N} \circ(\Lambda, E)^{\#} .
$$

DEFINITION 4. A strict Jacobi-Nijenhuis manifold $(M,(\Lambda, E), \mathcal{N})$ is a Jacobi manifold $(M, \Lambda, E)$ with a Nijenhuis operator $\mathcal{N}$ satisfying the following compatibility conditions: (i) $\mathcal{N} \circ(\Lambda, E)^{\#}=(\Lambda, E)^{\#} \circ{ }^{t} \mathcal{N}$ and (ii) $\mathcal{C}((\Lambda, E), \mathcal{N})=0$, where

$$
\begin{gathered}
\mathcal{C}((\Lambda, E), \mathcal{N})((\alpha, f),(\beta, h))=[(\alpha, f),(\beta, h)]_{\left(\Lambda_{1}, E_{1}\right)}-\left[{ }^{t} \mathcal{N}(\alpha, f),(\beta, h)\right]_{(\Lambda, E)} \\
-\left[(\alpha, f),{ }^{t} \mathcal{N}(\beta, h)\right]_{(\Lambda, E)}+{ }^{t} \mathcal{N}[(\alpha, f),(\beta, h)]_{(\Lambda, E)},
\end{gathered}
$$

for all $(\alpha, f),(\beta, h) \in \Omega^{1}(M) \times C^{\infty}(M),{ }^{t} \mathcal{N}$ is the transpose of $\mathcal{N}$ and $[,]_{(\Lambda, E)}$ (resp. $\left.[,]_{\left(\Lambda_{1}, E_{1}\right)}\right)$ is the bracket $(5)$ associated with $(\Lambda, E)$ (resp. $\left(\Lambda_{1}, E_{1}\right)$ ). 
For more details on (strict) Jacobi-Nijenhuis manifolds, see [12] and [14].

There exists a close relation between Poisson-Nijenhuis and strict Jacobi-Nijenhuis manifolds, as the next Proposition illustrates.

PROPOSITION 2. [14] With each strict Jacobi-Nijenhuis manifold $(M,(\Lambda, E), \mathcal{N})$, $\mathcal{N}:=(N, Y, \gamma, g)$, a Poisson-Nijenhuis manifold $(\tilde{M}, \tilde{\Lambda}, \tilde{N})$ can be associated, where $(\tilde{M}, \tilde{\Lambda})$ is the Poissonization of $(M, \Lambda, E)$, i.e. $\tilde{M}=M \times \mathbb{R}$ and $\tilde{\Lambda}=$ $e^{-t}\left(\Lambda+\frac{\partial}{\partial t} \wedge E\right)$, and $\tilde{N}$ is the Nijenhuis tensor field on $\tilde{M}$, given by $\tilde{N}=$ $N+Y \otimes d t+\frac{\partial}{\partial t} \otimes \gamma+g \frac{\partial}{\partial t} \otimes d t$, and conversely ( $t$ is the usual coordinate on $\mathbb{R}$ ).

Let us now consider a differentiable manifold equipped with a Nijenhuis operator $\mathcal{N}:=(N, Y, \gamma, g)$, given by (14). Using the operator $\mathcal{N}$, we may define a new bracket on $\mathfrak{X}(M) \times C^{\infty}(M)$, which is a deformation of the bracket (7), by setting, for all $(X, f),(Z, h) \in \mathfrak{X}(M) \times C^{\infty}(M)$,

$$
[(X, f),(Z, h)]_{\mathcal{N}}=[\mathcal{N}(X, f),(Z, h)]+[(X, f), \mathcal{N}(Z, h)]-\mathcal{N}[(X, f),(Z, h)] .
$$

Since the Nijenhuis torsion $\mathcal{T}(\mathcal{N})$ of $\mathcal{N}$ vanishes, the bracket [, $]_{\mathcal{N}}$ is a Lie bracket on $\mathfrak{X}(M) \times C^{\infty}(M)$ and $\left(T M \times \mathbb{R},[,]_{\mathcal{N}}, \pi \circ \mathcal{N}\right)$ is a Lie algebroid over $M$, where $\pi: T M \times \mathbb{R} \rightarrow T M$ is the projection over the first factor.

The differential of the Lie algebroid $\left(T M \times \mathbb{R},[,]_{\mathcal{N}}, \pi \circ \mathcal{N}\right)$ is $d_{\mathcal{N}}=\left[i_{\mathcal{N}}, \mathrm{d}\right]$, where [,] is the graded commutator, $\mathrm{d}=(d,-d)$ with $d$ the de Rham differential and $i_{\mathcal{N}}$ is the derivation of degree zero defined, for all $(\beta, \alpha) \in \Omega^{k}(M) \oplus \Omega^{k-1}(M)$, by

$$
\begin{gathered}
i_{\mathcal{N}}(\beta, \alpha)\left(\left(X_{1}, f_{1}\right), \cdots,\left(X_{k}, f_{k}\right)\right) \\
=\sum_{i=1}^{k}(\beta, \alpha)\left(\left(X_{1}, f_{1}\right), \cdots, \mathcal{N}\left(X_{i}, f_{i}\right), \cdots,\left(X_{k}, f_{k}\right)\right), \\
\quad\left(X_{1}, f_{1}\right), \cdots,\left(X_{k}, f_{k}\right) \in \mathfrak{X}(M) \times C^{\infty}(M) .
\end{gathered}
$$

PROPOSITION 3. The pair $(\gamma, g) \in \Omega^{1}(M) \times C^{\infty}(M)$ is a 1-cocycle of the Lie algebroid $\left(T M \times \mathbb{R},[,]_{\mathcal{N}}, \pi \circ \mathcal{N}\right)$.

Proof: Let $(X, f)$ and $(Z, h)$ be any sections of $\mathfrak{X}(M) \times C^{\infty}(M)$. A straightforward computation, using the fact that the Nijenhuis torsion of $\mathcal{N}$ is zero, leads to

$$
\begin{aligned}
(\gamma, g)\left([(X, f),(Z, h)]_{\mathcal{N}}\right)= & (N X+f Y) \cdot(\langle\gamma, Z\rangle+g h)-(N Z+h Y) \cdot(\langle\gamma, X\rangle+f g) \\
= & (\pi \circ \mathcal{N})(X, f) \cdot(\langle(\gamma, g),(Z, h)\rangle) \\
& -(\pi \circ \mathcal{N})(Z, h) \cdot(\langle(\gamma, g),(X, f)\rangle) .
\end{aligned}
$$

Note that $(\gamma, g)={ }^{t} \mathcal{N}(0,1)$.

Before giving our main theorem, we need to review some results from [2]. Given a Lie algebroid $(A,[],, \rho)$ over $M$, let us consider the vector bundle $\tilde{A}=A \times \mathbb{R} \rightarrow$ 
$M \times \mathbb{R}$ over $M \times \mathbb{R}$. The sections of $\tilde{A}$ can be identified with the $t$-dependent sections of $A, t$ being the canonical coordinate on $\mathbb{R}$, i.e. for any $\tilde{X} \in \Gamma(\tilde{A})$ and $(x, t) \in M \times \mathbb{R}, \tilde{X}(x, t)=\tilde{X}_{t}(x)$, where $\tilde{X}_{t} \in \Gamma(A)$. This identification induces, in a natural way, a Lie bracket on $\Gamma(\tilde{A})$, also denoted by $[$,$] :$

$$
[\tilde{X}, \tilde{Z}](x, t)=\left[\tilde{X}_{t}, \tilde{Z}_{t}\right](x), \quad \tilde{X}, \tilde{Z} \in \Gamma(\tilde{A}), \quad(x, t) \in M \times \mathbb{R},
$$

and a bundle map, also denoted by $\rho, \rho: \tilde{A} \rightarrow T(M \times \mathbb{R}) \equiv T M \oplus T \mathbb{R}$, in such a way that $(\tilde{A},[],, \rho)$ becomes a Lie algebroid over $M \times \mathbb{R}$.

Now, take a 1-cocycle $\theta \in \Gamma\left(A^{*}\right)$ and consider the following new brackets on $\Gamma(\tilde{A}):$

$$
[\tilde{X}, \tilde{Z}]^{\star \theta}=\exp (-t)\left([\tilde{X}, \tilde{Z}]+\theta(\tilde{X})\left(\frac{\partial \tilde{Z}}{\partial t}-\tilde{Z}\right)-\theta(\tilde{Z})\left(\frac{\partial \tilde{X}}{\partial t}-\tilde{X}\right)\right)
$$

and

$$
[\tilde{X}, \tilde{Z}]^{-\theta}=[\tilde{X}, \tilde{Z}]+\theta(\tilde{X}) \frac{\partial \tilde{Z}}{\partial t}-\theta(\tilde{Z}) \frac{\partial \tilde{X}}{\partial t},
$$

$\tilde{X}, \tilde{Z} \in \Gamma(\tilde{A})$. Also consider the maps $\rho^{\star \theta}, \rho^{-\theta}: \Gamma(\tilde{A}) \rightarrow \mathcal{V}^{1}(M \times \mathbb{R})$ given, for any $\tilde{X} \in \Gamma(\tilde{A})$, respectively by

$$
\rho^{\star \theta}(\tilde{X})=\exp (-t)\left(\rho(\tilde{X})+\theta(\tilde{X}) \frac{\partial}{\partial t}\right)
$$

and

$$
\rho^{-\theta}(\tilde{X})=\rho(\tilde{X})+\theta(\tilde{X}) \frac{\partial}{\partial t} .
$$

LEMMA 4. [2] Let $A \rightarrow M$ be a vector bundle over $M$, [, ] $: \Gamma(A) \times \Gamma(A) \rightarrow$ $\Gamma(A)$ a bracket on $\Gamma(A), \rho: \Gamma(A) \rightarrow \mathfrak{X}(M)$ a homomorphism of $C^{\infty}(M)$-modules and $\theta$ a section of the dual bundle $A^{*}$. Then the following conditions are equivalent:

(i) $(A,[],, \rho)$ is a Lie algebroid over $M$ and $\theta$ is a 1-cocycle,

(ii) $\left(\tilde{A},[,]^{\star \theta}, \rho^{\star \theta}\right)$ is a Lie algebroid over $M \times \mathbb{R}$,

(iii) $\left(\tilde{A},[,]^{-\theta}, \rho^{-\theta}\right)$ is a Lie algebroid over $M \times \mathbb{R}$.

LEMMA 5. [2] If $\left(\left(A \times \mathbb{R},[,]^{-\theta}, \rho^{-\theta}\right),\left(A^{*} \times \mathbb{R},[,]_{*}^{\star W}, \rho_{*}^{\star W}\right)\right)$ is a Lie bialgebroid (over $\tilde{M}=M \times \mathbb{R})$, then $\left((A, \theta),\left(A^{*}, W\right)\right)$ is a Jacobi bialgebroid (over $M$ ), and conversely.

THEOREM 6. Let $(M, \Lambda, E)$ be a Jacobi manifold and $\mathcal{N}=:(N, Y, \gamma, g)$ a Nijenhuis operator on $M$. Then $(M,(\Lambda, E), \mathcal{N})$ is a strict Jacobi-Nijenhuis manifold if and only if the pair

$$
\left(\left(\left(T M \times \mathbb{R},[,]_{\mathcal{N}}, \pi \circ \mathcal{N}\right),(\gamma, g)\right),\left(\left(T^{*} M \times \mathbb{R},[,]_{(\Lambda, E)}, \pi \circ(\Lambda, E)^{\sharp}\right),(-E, 0)\right)\right)
$$

is a Jacobi bialgebroid. 
Proof: From Proposition 2, $(M,(\Lambda, E), \mathcal{N})$ is a strict Jacobi-Nijenhuis manifold if and only if $(\tilde{M}, \tilde{\Lambda}, \tilde{N})$ is a Poisson-Nijenhuis manifold, which is equivalent to the fact that the pair $\left(\left(T \tilde{M},[,]_{\tilde{N}}, \tilde{N}\right),\left(T^{*} \tilde{M},[,]_{\tilde{\Lambda}}, \tilde{\Lambda}^{\sharp}\right)\right)$ is a Lie bialgebroid over $\tilde{M}=M \times \mathbb{R}$ (cf. Theorem 1$)$.

Now, using Lemma 4 and taking into account that the map

$$
\psi:\left(T \tilde{M},[,]_{\tilde{N}}, \tilde{N}\right) \rightarrow\left((T M \times \mathbb{R}) \times \mathbb{R},[,]_{\mathcal{N}}^{-(\gamma, g)},(\pi \circ \mathcal{N})^{-(\gamma, g)}\right),
$$

$\psi\left(\tilde{X}+\tilde{f} \frac{\partial}{\partial t}\right)=(\tilde{X}, \tilde{f})$, and its adjoint,

$$
\psi^{*}:\left(\left(T^{*} M \times \mathbb{R}\right) \times \mathbb{R},[,]_{(\Lambda, E)}^{\star(-E, 0)},\left(\pi \circ(\Lambda, E)^{\sharp}\right)^{\star(-E, 0)}\right) \rightarrow\left(T^{*} \tilde{M},[,]_{\tilde{\Lambda}}, \tilde{\Lambda}^{\sharp}\right),
$$

$\psi^{*}(\tilde{\alpha}, \tilde{f})=\tilde{\alpha}+\tilde{f} d t$, are Lie algebroid isomorphisms, we may conclude that

$\left(\left((T M \times \mathbb{R}) \times \mathbb{R},[,]_{\mathcal{N}}^{-(\gamma, g)},(\pi \circ \mathcal{N})^{-(\gamma, g)}\right),\left(\left(T^{*} M \times \mathbb{R}\right) \times \mathbb{R},[,]_{(\Lambda, E)}^{\star(-E, 0)},\left(\pi \circ(\Lambda, E)^{\sharp}\right)^{\star(-E, 0)}\right)\right)$

is a Lie bialgebroid over $\tilde{M}=M \times \mathbb{R}$ if and only if $(M,(\Lambda, E), \mathcal{N})$ is a strict Jacobi-Nijenhuis manifold. Finally, from Lemma 5 , we get the desired result.

PROPOSITION 7. The Jacobi structure induced on $M$ by the Jacobi bialgebroid $\left((T M \times \mathbb{R},(\gamma, g)),\left(\left(T^{*} M \times \mathbb{R},(-E, 0)\right)\right)\right.$ coincides with the one defined by $\left(\Lambda_{1}, E_{1}\right)^{*}$ $=\mathcal{N} \circ(\Lambda, E)^{\#}$.

Proof: Taking into account (6) and (13), and also the equality $\langle\gamma, E\rangle=0[14]$ we have, for all $f, h \in C^{\infty}(M)$,

$$
\begin{aligned}
\{f, h\} & =\left\langle d_{\mathcal{N}}^{(\gamma, g)} f, d_{*}^{(E, 0)} h\right\rangle \\
& =\left\langle d f,\left(-N \Lambda^{\sharp}+Y \otimes E\right) d h\right\rangle-h\langle d f, N E\rangle+f\left\langle d h, \Lambda^{\sharp}(\gamma)+g E\right\rangle .
\end{aligned}
$$

Since $N \Lambda^{\sharp}-Y \otimes E=\Lambda_{1}^{\sharp}$ and $\Lambda^{\sharp}(\gamma)+g E=N E=E_{1}$ (see [14]), the proof is complete.

A natural question that arises is the following: can we also establish, for the weak Poisson-Nijenhuis manifolds and for the Jacobi-Nijenhuis manifolds, a similar characterization, using the Lie algebroids theory? We postpone the answer for a subsequent paper.

\section{REFERENCES}

[1] J. Grabowski and G. Marmo: Preprint (2001), arXiv:math.DG/0111148.

[2] D. Iglesias and J. C. Marrero: J. Geom. Phys. 40 (2001), 176-200.

[3] D. Iglesias-Ponte and J. C. Marrero: Extracta Mathematicae (2002), to appear.

[4] M. de León, J. C. Marrero and E. Padrón: C. R. Acad. Sci. Paris 325 Série I (1997), 405-410.

[5] Y. Kerbrat and Z. Souici-Benhammadi: C. R. Acad. Sci. Paris 317 Série I (1993), 81-86.

[6] Y. Kosmann-Schwarzbach: Acta Appl. Math. 41 (1995), 153-165.

[7] Y. Kosmann-Schwarzbach: Lett. Math. Phys. 38 (1996), 421-428. 
[8] Y. Kosmann-Schwarzbach and F. Magri: Ann. Inst. Henri Poincaré A 53 (1990), 35-81.

[9] K. Mackenzie: Lie groupoids and Lie algebroids in differential geometry, Cambridge University Press, 1987.

[10] K. Mackenzie and P. Xu: Duke Math. J. 73 (1994), 415-452.

[11] F. Magri and C. Morosi: A geometric characterization of integrable Hamiltonian systems through the theory of Poisson-Nijenhuis manifolds, Università di Milano, Quaderno S 19, 1984.

[12] J. C. Marrero, J. Monterde and E. Padrón: C. R. Acad. Sci. Paris 329 Série I (1999), 797-802.

[13] J. M. Nunes da Costa and C.-M. Marle: Diff. Geometry and its Appl. (Brno 1995), Masaryk Univ. Brno, 523-538, 1996.

[14] F. Petalidou and J. M. Nunes da Costa: J. Geom. Phys. (2002), to appear. 